\title{
El simposio de Königsberg sobre fundamentos de la matemática en perspectiva*
}

The Königsberg's Symposium on Foundations of Mathematics in Perspective

Oscar M. Esquisabel

Javier Legris ${ }^{\ddagger}$

\begin{abstract}
Resumen
Este volumen de Metatheoria incluye traducciones al castellano de los tres famosos trabajos sobre las escuelas de fundamentos de la matemática, el logicismo, el intuicionismo y el formalismo, expuestos en el Simposio de Königsberg sobre Fundamentos de la Matemática en septiembre de 1930, y que fueron finalmente publicados en la revista Erkenntnis en 1931. Los tres constituyeron un hito en la filosofía de la matemática del siglo pasado. En esta introducción a las traducciones, los compiladores del volumen esbozan el contexto histórico en el cual se concibieron los trabajos originales e incluyen detalles de su publicación original. También ponen de relieve el papel decisivo que tuvieron en la filosofía de la matemática del siglo pasado. Se hace un resumen de sus contenidos, y se discuten las ideas subyacentes del Círculo de Viena acerca de la naturaleza de la matemática y el impacto de los teoremas de Gödel en los programas de fundamentos. Además, se hace una breve introducción a las contribuciones originales que siguen en el volumen a las traducciones.
\end{abstract}

Palabras clave: fundamentos de la matemática, filosofía de la matemática del siglo XX, Círculo de Viena, teoremas de Gödel

\begin{abstract}
This volume of Metatheoria includes translations into Spanish of the three famous papers on the schools in foundations of mathematics, logicism, intuitionism and formalism, presented at the Königsberg's Symposium on Foundations of Mathematics in September 1930 and finally published in the journal Erkenntnis in 1931. The three papers constituted a milestone in the Philosophy of Mathematics of the last century. In this introduction to the translations, the editors of the volume outline the historical context in which the original papers were conceived and they include some details concerning their original publication. They also stress the decisive role played by the papers in the philosophy of mathematics in the last century. A summary of them is provided and the underlying ideas of the Vienna Circle on the nature of mathematics and the impact of Gödel theorems in the foundations programs are discussed. Moreover, they briefly introduce the original contributions to the volume that follow the translations.
\end{abstract}

Keywords: foundations of mathematics, 20th century philosophy of mathematics, Vienna Circle, Gödel theorems

\footnotetext{
* Recibido: 14 de septiembre de 2018. Aceptado con revisiones: 20 de octubre de 2018.

† CONICET, Universidad Nacional de La Plata, Argentina. Para contactar al autor, por favor, escribir a: omesqui@gmail.com.

$\$$ CONICET, Universidad de Buenos Aires, IIEP-BAIRES, Argentina. Para contactar al autor, por favor, escribir a: Javier.Legris@fce.uba.ar.

Metatheoria 10(2)(2020): 7-15. ISSN 1853-2322. eISSN 1853-2330.

(C) Editorial de la Universidad Nacional de Tres de Febrero.

(C) Editorial de la Universidad Nacional de Quilmes.

Publicado en la República Argentina.
} 
En septiembre de 1930 tuvieron lugar en la ciudad de Königsberg (capital en ese entonces de Prusia Oriental y actualmente la ciudad rusa de Kaliningrado) cuatro congresos científicos simultáneamente. Entre ellos se contaban las jornadas anuales de la Deutscher Mathematiker Vereiningung (DMV) y las "Segundas Jornadas de Epistemología de las Ciencias Exactas" (“2. Tagung für Erkenntnislehre der exakten Wissenschaften") que se desarrollaron del 5 al 7 de septiembre (las primeras habían tenido lugar en Praga el año anterior). Estas actividades dejaron su marca en la historia de la matemática. Por de pronto, en la ocasión David Hilbert ofreció su célebre alocución radial (cuya grabación se conserva, ver: https://www.youtube.com/watch?v=EbgAu_X2mm4). La alocución era una versión abreviada de su conferencia "Conocimiento de la naturaleza y lógica".

Pero además, las segundas jornadas dedicadas a la epistemología de las ciencias exactas hicieron historia en la filosofía de la matemática, sobre todo porque en ellas tuvo lugar un simposio dedicado a la situación en los fundamentos de la matemática, que en ese momento se manifestaba a través de las "tres grandes escuelas" u orientaciones: el logicismo, el intuicionismo y el formalismo. Esta tripartición determinó durante décadas la investigación en los fundamentos y la discusión en la filosofía de la matemática. La mayoría de la bibliografía sobre el tema mencionaba al menos a alguna de las tres escuelas, y era usual en los libros introductorios incluir una exposición de las tres. A partir de las paradojas ligadas a la teoría de conjuntos descubiertas a fines del siglo XIX, se generó una amplia y rica discusión acerca de cuáles debían ser las "bases sólidas" (esto es, que aseguraran consistencia) sobre las cuales fundar la matemática. Es en este contexto que se perfilaron las tres escuelas como soluciones alternativas al problema. En una conferencia ofrecida en Zúrich en 1920, Hermann Weyl introdujo la expresión "crisis de fundamentos" (Grundlagenkrise) explotando algunas analogías con los problemas de la política europea en esa época: las célebres paradojas parecían ser tan solo "escaramuzas" en las "fronteras" del reino de la matemática, pero cabía el peligro de que la "solidez y seguridad" del "reino" se viera afectada en su centro mismo (ver Weyl 1921).

La expresión se instaló entre los filósofos y matemáticos, indicando una situación insostenible que exigía una solución. De hecho, esta pretendida crisis tuvo consecuencias tanto en la matemática como en la filosofía. En la primera, triunfó la idea de una base conceptual y abstracta que se manifestó en la teoría de conjuntos y también en la idea bourbakiana de basar la matemática en tipos de estructuras. En la segunda, el positivismo lógico y la filosofía analítica pudieron poner a prueba sus ideas y su metodología. No obstante, el resultado tal vez más importante fue la consolidación de la lógica matemática como disciplina independiente (tal como se pone de relieve en el trabajo de Lassalle Casanave y Pereira incluido en este volumen).

El simposio tuvo como eje tres conferencias en alemán dedicadas a cada una de las tres escuelas dictadas respectivamente por Rudolf Carnap (logicismo), Arendt Heyting (intuicionismo) y Johann von Neumann (programa de Hilbert o "formalismo"). Los conferencistas habrían sido propuestos por Carnap, quien le había ofrecido a Hans Hahn hablar sobre logicismo, pero este no aceptó por razones meramente coyunturales. Como asistente del simposio se encontraba también el joven y recién doctorado Kurt Gödel, quien sorprendió a todos con una breve y tímida comunicación acerca de su célebre teorema de incompletitud de la aritmética formalizada.

El miércoles 3 de septiembre Carnap se encontró en Berlín con Gödel, Herbert Feigl y Frederich Waismann, procedentes de Viena, y el grupo partió en tren hacia la ciudad de Swinemünde en el Mar Báltico (actual Świnoujście en Polonia), donde se les unieron Kurt Grelling y Hans Hahn. El grupo partió desde allí en barco hacia Königsberg, rodeando la costa polaca (ver Köhler 1991, p. 152). El día 5 tuvieron lugar las tres conferencias centrales que llevaron respectivamente por título "Die Grundgedanken des Logizismus", "Die intuitionistische Begründung der Mathematik" y "Die axiomatische Begründung der Mathematik". A estas siguió una presentación de Friedrich Waismann con el título "Das Wesen der Mathematik: der Standpunkt Wittgensteins", que fue agregada a último momento. El contenido de las tres conferencias fue publicado, con títulos ligeramente diferentes y unificados, en el volumen 2 de la revista Erkenntnis de 1932, siendo estas las versiones que nos han llegado y que aparecen traducidas en este volumen. Es interesante notar que la charla de Waismann nunca fue publicada, tal vez como consecuencia de la observación hecha por Carnap de que las 
"interesantes ideas" de Wittgenstein debían ser expuestas "de una forma madura para la discusión”. Al día siguiente expusieron Hans Reichenbach, Werner Heisenberg y Otto Neugebauer y a continuación hicieron presentaciones breves Gödel (sobre su teorema de completitud para la lógica de los Principia), Arnold Scholz y Walter Dubislav. La presentación de Neugebauer, que años antes se había doctorado en Göttingen sobre la aritmética del antiguo Egipto y había comenzado a trabajar sobre la matemática babilónica, tenía por título "Zur vorgriechische Mathematik" y fue publicada en el mismo volumen de Erkenntnis.

El último día del simposio estuvo dedicado a una discusión que aspiraba a aclarar diferentes puntos de las conferencias dedicadas al problema de los fundamentos, pero que también incluyó algunas aportaciones independientes (Hahn et al. 1931). La discusión fue coordinada por Hans Hahn y en ella participaron, además de los tres conferencistas, Scholz, Reidemeister y Gödel. Hahn abrió la discusión con una defensa del logicismo partiendo de un punto de vista empirista acerca de la aplicabilidad de la lógica y la matemática a lo real; para Hahn es precisamente esta aplicabilidad lo que debe fundamentarse (Hahn et al. 1931, p. 35 y ss.). Aquí resuenan ideas de la época temprana del Círculo de Viena (lo que se ha llamado "Primer Círculo de Viena", ver Haller 1986), cuyos miembros estaban especialmente preocupados por la aplicación de la matemática a las ciencias empíricas. Sobre la base del carácter tautológico de la lógica, el logicismo lleva a concluir, de un modo que para Hahn es compatible con el empirismo, que la matemática "no dice nada acerca de la realidad". No obstante, Hahn reconoce las dificultades en justificar la reducción a la lógica del principio de inducción completa o del axioma de elección (Hahn et al. 1931, p. 138). La exposición de Hahn representaba claramente, con sus luces y sombras, la discusión de estos problemas en el Círculo de Viena y la búsqueda de una solución combinando aspectos del logicismo con el formalismo de Hilbert.

En su intervención Gödel se dedica a señalar las dificultades en el formalismo de Hilbert que se siguen de su teorema de incompletitud. Así, señala: "Nunca podemos estar seguros de que todas las consideraciones sobre el contenido sean representables en un sistema formal determinado" (Gödel 2006, p. 90). Gödel recurre al caso de enunciados indecidibles, como la conjetura de Goldbach, los que "pueden ser verdaderos en cuanto a su contenido, pero no son deducibles en el sistema formal de la matemática clásica” (Gödel 2006, p. 91). Afirmaciones como esta seguirán siendo discutidas en los años posteriores, en el contexto del intuicionismo y de la teoría de la demostración. Gödel también menciona el teorema de Fermat como caso de enunciado indecidible, el cual, como es sabido, fue demostrado utilizando métodos analíticos, hace unos pocos años. La versión publicada incluye, además, un "apéndice" con una breve presentación del teorema de incompletitud hecha por Gödel especialmente para la ocasión (Gödel 2006, pp. 91-93).

Las tres conferencias llegaron a constituir una especie de "manifiesto" (siguiendo la expresión de John Dawson, ver Dawson 1984) de las tres corrientes. Una muestra del peso que tuvieron lo da el hecho de que la primera parte de la notable compilación realizada por Paul Benacerraf y Hilary Putnam, Philosophy of Mathematics. Selected Readings, publicada en 1963, contiene traducciones al inglés de las tres conferencias, las que fueron también incluidas en la muy modificada segunda edición de 1983. Según los compiladores las escuelas constituían "las tres concepciones tradicionalmente importantes acerca de la naturaleza de la matemática” (Benacerraf \& Putnam 1983, p. 1). Pese a la evolución que ha experimentado la filosofía de la matemática desde entonces, las tres escuelas forman parte de su contenido tradicional (un caso clásico es el libro de Stephan Körner Philosophy of Mathematics: An Introductory Essay, reimpreso por la editorial Dover y traducido al castellano), y también en obras bastante recientes (ver, por ejemplo, Bostock 2009 y el artículo dedicado a la filosofía de la matemática en el Continuum Companion to Philosophy of Science, Pincock 2011).

Los trabajos del simposio discuten las respectivas escuelas a partir de perspectivas específicas. El logicismo en la matemática puede resumirse en la bien conocida tesis de que toda la matemática se reduce a la lógica, de modo que todas las verdades matemáticas no son más que verdades lógicas. Esta tesis fue planteada por Gottlob Frege, si bien limitada exclusivamente al caso de la aritmética. El programa de fundamentación del logicismo aparece ya en su obra Begriffsschrift (Notación conceptual) de 
1879, pero es en su libro Die Grundlagen der Arithmetik (Los fundamentos de la aritmética) publicado en 1884, donde Frege se dedica a desarrollar las ideas que subyacen al programa y también a criticar tanto la posición de Kant, para quien los enunciados matemáticos eran sintéticos a priori, como la posición empirista, muy difundida en ese momento, adjudicada a John Stuart Mill, quien entendía las leyes aritméticas como leyes empiricas generalizadas, leyes que era en definitiva psicológicas. La reducción de la aritmética a la lógica dependía, en el sistema de Frege, de la consideración de las extensiones de conceptos (que pueden interpretarse alternativamente en términos de conjuntos) como entidades lógicas. En definitiva, el logicismo resulta ser una manera de justificar el carácter analítico y a priori de la matemática, constituyendo una variante de racionalismo respecto de las ciencias formales. Bertrand Russell mostró en 1903 una inconsistencia en el programa logicista de Frege, la famosa "paradoja de Russell" relativa al "conjunto de todos los conjuntos". Esta paradoja fue precisamente la primera manifestación de lo que se llamó posteriormente "crisis de fundamentos".

El trabajo de Carnap tiene más en cuenta la presentación que hicieron Alfred North Withehead y Russell en la obra Principia Mathematica, donde se resuelve la inconsistencia mencionada por medio de la teoría de tipos. Carnap era sin duda un logicista muy atípico. En el momento de la presentación en Königsberg, estaba luchando con su propio proyecto "monolingüístico" para desarrollar una metateoría de la lógica, que tendría como producto final la "sintaxis lógica" (para más detalles ver inter alia Legris 1996). En una carta a von Neumann del 11 de julio de 1931, Carnap afirmaba que su propia concepción de los fundamentos de la matemática "se basaba en una combinación de las ideas de Russell y de Hilbert" (citado en Mancosu 1999, p. 41). En este punto cabe recordar que Hilbert ya había manifestado inclinaciones logicistas (como señalan Fernández de Castro y Torres Falcón en su contribución a este volumen). Por lo demás, se pueden percibir en Carnap ciertos indicios de lo que será la "herencia oculta" del logicismo (para usar la expresión del trabajo de Ferreirós publicado en este volumen).

El intuicionismo tiene una clara fecha de nacimiento en la tesis de doctorado que en 1907 presentó Luitzen Egbertus Jan Brouwer en la universidad de Amsterdam, y que llevaba el título Sobre los fundamentos de la matemática. La tesis central de Brouwer era que la matemática surge de la actividad mental del sujeto en el tiempo. A esta actividad mental (que prima facie no debe tomarse en un sentido psicológico) la considera una intuición, y de ahí el nombre que recibió la escuela. En realidad, esta actividad mental se rige por operaciones que construyen las entidades matemáticas, de aquí que es mejor hablar de una forma de constructivismo. El intuicionismo fue de las tres la escuela más heterodoxa y la que generó mayor controversia (con entusiasmo Weyl declaraba: “ßBrouwer es la revolución!”). Arendt Heyting fue tal vez el discípulo más sobresaliente de Brouwer y fue quien se dedicó con más ahínco a sistematizar las ideas del maestro y difundirlas en los medios filosóficos y matemáticos. Contrariamente a Brouwer, Heyting dedicó una parte importante de su obra a la formalización de la lógica intuicionista y presentarla de acuerdo con la metodología usual de la lógica matemática. De este modo, Heyting formuló sendos sistemas axiomáticos para la lógica y la aritmética intuicionistas, y también esbozó una semántica basada en la idea de demostración constructiva, abriendo una rica perspectiva de análisis sobre la que se continúa trabajando en la actualidad (y que, como se indica en el trabajo de Lassalle Casanave y Pereira incluido en este volumen, dio frutos en la teoría de la demostración). El trabajo de Heyting es un ejemplo temprano de esta perspectiva y en él pueden apreciarse las raíces de la aproximación semántica al intuicionismo, siendo uno de sus textos fundamentales.

El concepto de "formalismo" ha sido entendido de muy diferentes maneras en la historia de la filosofía de la matemática. En el contexto de la contribución de von Neumann el concepto se aplica, de manera específica, al "programa de Hilbert", a saber, el programa de fundamentación que David Hilbert concibió y comenzó a desarrollar junto con su equipo de colaboradores (sobre todo Paul Bernays quien tenía además formación filosófica) en la universidad de Göttingen alrededor de 1920. Esta era una importante y ambiciosa empresa de reconstrucción de la matemática cuyo objetivo puede resumirse en demostrar la consistencia (esto es, la ausencia de contradicciones) en las teorías más básicas de la matemática. Para ello debía haber una "base incuestionable" a partir de la cual llevar a cabo tales 
demostraciones de consistencia, y Hilbert veía esta base en ciertos procedimientos muy elementales empleados en la construcción y manipulación de signos. Hilbert llamaba a estos procedimientos "finitos" y la sistematización de los mismos dio lugar a la disciplina que Hilbert bautizó como "metamatemática". Von Neumann finaliza su trabajo señalando como tarea para el futuro la demostración por medios finitos de la consistencia de la aritmética en su totalidad.

Benacerraf y Putnam observan que en las tres corrientes el concepto de infinito (el de conjunto infinito, si se quiere ser más preciso) juega un papel fundamental (Benacerraf \& Putnam 1983, p. 6). Desde luego, este concepto continúa siendo problemático desde el punto de vista filosófico (basta con mencionar el problema del infinito en la práctica matemática o la evaluación del análisis no estándar en relación con la concepción conjuntista de los números reales). De este modo se encuentra aquí una razón para seguir buceando en los argumentos planteados en los textos clásicos de Frege, Russell, Brouwer, Heyting, Bernays, Hilbert y, por supuesto, en los tres trabajos que se presentan traducidos al español en este volumen. En las tres corrientes aparecen argumentos que remiten a las tradicionales posiciones de platonismo, constructivismo y nominalismo, según el caso, y también aparecen afirmaciones que hacen pensar en posturas pragmatistas o naturalistas, que son importantes en la discusión actual. Muchas observaciones gnoseológicas (como las relativas a la intuición o a la demostración matemática) son formuladas actualmente desde las perspectivas de las ciencias cognitivas.

El anuncio que en el mismo simposio hizo Gödel acerca de su resultado de incompletitud provocó un enorme alboroto entre los asistentes y llevó a considerar el programa de Hilbert como una causa perdida. En una carta escrita a Carnap, del 7 de junio de 1931, von Neumann afirmó la "irrealizabilidad" (Undurchführbarkeit) del programa de Hilbert, y sostenía que, aunque no había razones para rechazar el intuicionismo, la discusión sostenida en Königsberg estaba "superada” (überholt). En el semestre de invierno 1930/1931 von Neumann ofreció en Berlín un seminario sobre la Beweistheorie de Hilbert. Según el testimonio de Carl Hempel, asistente al seminario, von Neumann recibió una carta de Gödel con la demostración detallada de su teorema de incompletitud, llevándolo a abandonar el tema y dedicarse exclusivamente a la presentación del teorema (ver Hempel 2000, p. 14). Al mismo tiempo, el coloquio que Hans Reichenbach coordinaba en Berlín le habría dedicado una sesión especial al teorema en diciembre de 1930, a escasos tres meses después del simposio (ver Mancosu 1999, p. 35).

Es sabido que Gödel no opinaba que su resultado echara enteramente por tierra el programa de Hilbert; tampoco sostenía que afectara directamente a la matemática intuicionista. En los protocolos de la reunión del círculo de Schlick en Viena el 15 de enero de 1931, consta que Gödel, respondiendo a una objeción de Felix Kauffmann, remarcó que su resultado no tiene nada que ver con una demostración de consistencia "en el sentido del pensamiento con contenido" ("im Sinne inhaltlichen Denkens”, citado en Mancosu 1999, p. 37 y ss.). Según cuenta Constance Reid, Hilbert se enteró del resultado de Gödel a través de Bernays y, si bien se sintió contrariado y frustrado al principio, luego intentó adaptar su programa a la nueva situación (ver Reid 1970, p. 198). Esta actitud condujo a un relajamiento de la caracterización del concepto de método finito, que adoptó diferentes estrategias, examinadas en el segundo volumen de los Grundlagen der Mathematik de Hilbert y Bernays publicado en 1938 (un resumen de estas estrategias puede encontrarse en Raggio 1990). Una de estas estrategias fue la que, hacia 1935, siguió Gerhard Gentzen al considerar como "constructivas" ciertas formas de inducción transfinita. (Vale observar que, siendo estudiante en Berlín por un tiempo, Gentzen fue uno de los asistentes al seminario de von Neumann mencionado antes.) Sin duda, las ideas intuicionistas encontraron su oportunidad en el medio del escándalo producido por los teoremas de Gödel.

En suma, el simposio dejó una fuerte impronta en la historia de la filosofía de la matemática. Sus tres trabajos centrales han pasado a ser obras clásicas de las que vale la pena disponer en nuestra lengua. Las traducciones proceden de los originales en alemán, tal como aparecen en el volumen de Erkenntnis y se han agregado algunas notas aclaratorias donde correspondiera. Los traductores han consultado también las traducciones al inglés publicadas en Benacerraf y Putnam (1963), optando por versiones alternativas de algunas expresiones y señalando en notas algunas erratas. 
Los artículos publicados a continuación de las traducciones han sido escritos originalmente para este volumen, y tienen por objetivo iluminar el problema de los fundamentos de la matemática desde la perspectiva actual, mostrando rupturas y continuidades. También muestran algunos desarrollos y transformaciones que experimentaron las tres escuelas con posterioridad a 1931, dando evidencia del indiscutible peso en el desarrollo de la lógica simbólica y la filosofía de la matemática. En su contribución "La herencia oscura del logicismo", José Ferreirós se propone analizar la influencia más o menos encubierta del logicismo en el desarrollo de la lógica de la segunda mitad del siglo XX. En concreto, el autor sostiene la tesis de que tanto la semántica conjuntista para la lógica de segundo orden (LSO) como el desarrollo de la teoría de modelos a partir de Tarski revelan, de una u otra manera, conexiones profundas y poco exploradas con el logicismo clásico, por lo que pueden considerarse como su "herencia oscura". Como una forma de herencia explícita, Ferreirós aborda sintéticamente el neologicismo, no sin plantear algunas reservas acerca de su factibilidad.

El núcleo del trabajo está constituido por tres secciones. La primera de ellas analiza y critica los aspectos semánticos de la LSO, mientras que la segunda establece nexos entre el logicismo y los comienzos de la teoría de modelos con Tarski. Finalmente, en la tercera parte, se realiza una evaluación acerca de los alcances y límites de la LSO, teniendo en vista sus aplicaciones en el tratamiento de cuestiones filosóficas. De esta manera, en lo que respecta al primer problema, Ferreirós parte del hecho de que, inicialmente, los lógicos y matemáticos admitían la LSO como su instrumento natural de trabajo. Esto fue así debido a que, por ejemplo, Hilbert, Church y Ackermann, reconocían que la lógica de primer orden (LPO) era inadecuada para expresar nociones matemáticas y conjuntistas, para lo cual se requería la LSO. Así, la aplicación de la LSO parece proseguir con la tarea del logicismo en el sentido de expresar con recursos puramente lógicos el pensamiento matemático "real". Esta idea se refuerza por la posibilidad de demostrar en LSO la categoricidad de los axiomas de $\mathbb{N}$ y de $\mathbb{R}$, así como también la de probar la cuasi-categoricidad de $\mathrm{V}$ en el sistema axiomático para teoría de conjuntos (Zermelo 1930). Sea como fuere, Ferreirós apunta sus críticas precisamente a la conclusión de que este empleo de la LSO en la filosofía de la matemática esté exenta de supuestos extralógicos. Por otra parte, dichos supuestos tienen su origen en la tesis logicista de que la lógica se solapa con la teoría de conjuntos. Como resultado de ello, se llega a una semántica "plena” para LSO que implica la admisión de objetos incontrolables, impropios de la lógica.

En cuanto al logicismo y la teoría de modelos, Ferreirós aborda las orientaciónes inicialmente logicistas de Tarski, el fundador de la teoría de modelos. Es así que hacia 1940 Tarski produce un cambio de perspectiva en la metamatemática, mediante la adopción de la teoría de conjuntos y, por esta vía, de todos los métodos de la matemática clásica como recursos metateóricos. De este modo, el autor establece una conexión entre dicho giro con la proximidad inicial de Tarski a posturas logicistas, tal como se revela, por ejemplo en su libro Introduction to Logic, de 1940. En este sentido, Ferreirós califica la posición inicial de Tarski en términos de un logicismo "débil", al tiempo que interpreta sus desarrollos metamatemáticos como el resultado de una nueva comprensión de la matemática en términos de axiomatización y estructuras conjuntísticas, la cual tuvo lugar hacia 1930, aproximadamente.

Finalmente, Ferreirós realiza una evaluación de la interpretación estándar de la LSO. Desde su punto de vista, dicha interpretación es de carácter fuertemente matemático, por su dependencia de la noción de conjunto arbitrario y por el supuesto del dominio de todos los subconjuntos. A fin de eliminar los supuestos extralógicos, propone realizar una restricción de la LSO como sistema definido por reglas, con una semántica también restringida, del tipo propuesto por Leon Henkin. Según Ferreirós, es necesario elucidar los supuestos implícitos en la LSO estándar, así como en otros tipos de lógica, en sus aplicaciones a cuestiones filosóficas, puesto que nos comprometen con postulados ontológicos realistas muy fuertes.

En el trabajo "La constitución del programa de Hilbert", Max Fernández de Castro y Yolanda Torres Falción se proponen presentar una visión panorámica de la evolución de las ideas de Hilbert acerca de la fundamentación de la matemática, especialmente a la luz de las notas y cursos de Hilbert que han sido recientemente publicados. De esta forma, el trabajo se divide en dos partes, la primera de 
las cuales se dedica a las primeras investigaciones y trabajos hilbertianos acerca del método axiomático y a la discusión que Hilbert mantuvo con Frege acerca de la naturaleza de lo matemático. En la segunda parte, se analiza el surgimiento y desarrollo del programa metamatemático, especialmente en la década de 1920, hasta 1930, aproximadamente.

De este modo, se sintetiza la nueva concepción del método axiomático que implicó la axiomatización de la geometría propuesta por Hilbert en 1899 y se la confronta con la versión clásica de las propiedades y funciones de los sistemas axiomáticos. En ese marco, se reseñan los puntos fundamentales de la discusión que mantuvo Hilbert con Frege acerca de la naturaleza de los axiomas matemáticos y de la existencia matemática. En ese sentido, se señala que la axiomática 'formal' constituyó para Hilbert un método para determinar el objeto de estudio de la matemática. Esta manera de comprender la matemática proporciona la base para considerar a Hilbert como un precursor de un estructuralismo de tipo eliminativista. El trabajo dilucida la cuestión de la posible adhesión de Hilbert al logicismo, la cual, siguiendo los trabajos de Sieg y Ferreirós, se extendería hasta 1920, año de las primeras formulaciones del programa formalista. La respuesta a esta pregunta es sencilla, ya que dicha adhesión muestra avances y retrocesos, especialmente en lo que se refiere a los requisitos exigibles para la aceptación de conjuntos infinitos. Esta primera parte, concluye con un breve examen de la conferencia de Hilbert de 1917, "El pensamiento axiomático", donde retoma la cuestión de la consistencia de los sistemas axiomáticos y relativiza las pruebas relativas de consistencia.

La segunda parte del artículo comienza con una reseña de las investigaciones de Hilbert a partir del año 1917 y señala el año 1920 como la fecha del inicio del programa metamatemático, que se desarrolla de acuerdo con tres etapas sucesivas, perceptibles en las notas de ese mismo año, a saber, el rechazo definitivo del logicismo, la fundamentación de la matemática en un finitismo radical y, finalmente, el programa formalista propiamente dicho, que retoma ideas ya propuestas en 1905, consistentes en la formalización axiomática completa de una teoría, con el fin de procurar una prueba de consistencia absoluta. A partir de esta primera formulación del programa, la exposición acompaña el desarrollo de las ideas de Hilbert siguiendo el hilo conductor de sus publicaciones y conferencias. Así, entre los años 1922 y 1927 se destaca la adopción de diferentes formas de finitismo. De este modo, en "La nueva fundamentación de la matemática" (1922), a la par de una crítica de otras formas de "finitismo" como el de Weyl y Brouwer, propone el programa formalista basado en la certeza de los signos como objetos de la teoría de los números. Hacia 1923, reconoce la imposibilidad de reconstruir la totalidad de la matemática sobre las bases intuitivas que procuran los signos y retoma la necesidad de axiomatizar para proceder luego a una prueba de consistencia: se llega así al tercer estadio esbozado en las notas de 1920. A continuación, se pasa revista a la presentación del programa a través de diversas conferencias y obras, entre las que se destaca la tesis doctoral de Wilhelm Ackermann de 1924, realizada con la dirección de Bernays, donde presenta una prueba de consistencia para la aritmética recursiva primitiva. En 1925, Hilbert pronuncia su conferencia sobre el infinito, en la que expone una versión perfeccionada del programa de fundamentación, uno de cuyos puntos más importantes es la propuesta de extender una teoría mediante elementos ideales, a través de una prueba de consistencia finitaria. Finalmente, en el año 1927 Hilbert publica una descripción completa del programa formalista, en la que se retoman temas previamente abordados: la diferencia entre razonamiento formal y razonamiento de contenido, la insuficiencia de la lógica, la necesidad de recurrir a los signos como objetos concretos, la necesidad de una formalización completa de la matemática en la teoría de la demostración y la diferencia entre aritmética elemental y álgebra, cuyas expresiones constituyen objetos formales, demostrables por medio de reglas sintácticas. Como colofón del decurso del desarrollo del programa formalista, el trabajo evalúa el tipo de finitismo que defendió Hilbert especialmente en relación con el intuicionismo. La conclusión de los autores es que es difícil sostener que haya coincidencias plenas entre ambos tipos de finitismo, dado que las exigencias que en general los intuicionistas imponen al pensamiento matemático, para Hilbert sólo son aplicables en el plano metamatemático. Para terminar, se reseña la importancia que tuvieron los teoremas de incompletitud y el desarrollo de la teoría de funciones recursivas para la evolución posterior del programa formalista. 
En "A propósito del formalismo de Johann von Neumann", Lassalle Casanave y Pereira llevan a cabo tanto un análisis como una evaluación de las ideas de von Neumann acerca de la cuestión de los fundamentos de la matemática, con el trasfondo de su contribución al simposio de Königsberg. En la primera sección del artículo, los autores abordan el problema general de probar la confiablidad de la matemática. De este modo, von Neumann reduce un problema epistemológico y filosófico al problema matemático de demostrar la consistencia de la aritmética clásica, de acuerdo con métodos que sean aceptables para la aritmética intuicionista y en términos del lenguaje simbólico que proporcionaron los Principia Mathematica tanto para la lógica como para la matemática. Por esta vía, una demostración matemática se transforma en una secuencia de fórmulas de un lenguaje formal, que, a su vez, puede tratarse como un objeto matemático. Así, la demostración de consistencia de la matemática clásica se reduce a la demostración, por métodos finitarios, del hecho de que la secuencia de fórmulas del lenguaje formal no tiene una contradicción como fórmula final. En síntesis, tal es la tarea de la teoría de la demostración como ya la propone Hilbert en 1922. No obstante, el intento de reducción de la cuestión epistemológica relativa a la confiabilidad de la matemática en términos de la manipulación simbólica de un sistema formal requiere, por su parte, una justificación de cuya necesidad von Neumann no parece ser plenamente consciente. Al respecto, los autores establecen una clara distinción entre el formalismo metodológico y el sustantivo y recuerdan que, para Hilbert, la matemática es un juego simbólico sólo desde el punto de vista metodológico, es decir, metamatemático. En definitiva, el programa del formalismo, en términos de von Neumann, consiste mostrar la confiabilidad de la matemática no mediante el análisis finitario de los enunciados matemáticos considerados aisladamente, sino a través del control formal de los métodos de demostración de los que resultan.

La segunda sección del trabajo aborda las tareas que debe emprender una teoría de la demostración, a saber, la formalización y la demostración de consistencia. Para la cuestión de la formalización, los autores parten del trabajo de von Neumann de 1927 dedicado a este problema. En cuanto a la cuestión de la consistencia, el trabajo reconstruye la argumentación de von Neumann desde el punto de vista de la propiedad de conservatividad, en el sentido de que se hace necesario recurrir a la distinción entre enunciados ideales y reales. Por último, en la tercera sección se lleva a cabo una evaluación del programa formalista, especialmente en vista de los resultados de Gödel, que, como ya se comentó, se dieron a conocer paradójicamente durante el desarrollo del simposio y cuyos efectos para el programa formalista von Neumann reconoció en su plena dimensión. Finalmente, los autores abordan los esfuerzos de Gerhard Gentzen por superar las dificultades que resultan del teorema de Gödel y que se cristalizan en cinco demostraciones de la consistencia de la aritmética clásica, la primera de las cuales se remonta a 1933. Como conclusión, se retoma una de las hipótesis centrales del trabajo, a saber, que no está claro en qué medida el tratamiento de un problema epistemológico y filosófico puede resolverse mediante su reducción a planteamientos lógico-matemáticos, al tiempo que se destaca la supervivencia de la teoría de la demostración, gracias a los trabajos de Gentzen, como la herencia fundamental del programa formalista.

\section{Bibliografía}

Benacerraf, P. y H. Putnam (ed.) (1964), Philosophy of Mathematics: Selected Readings, New York: Prentice-Hall, $2^{\mathrm{a}}$ ed., Cambridge: Cambridge University Press, 1983.

Bostock, D. (2009), Philosophy of Mathematics. An Introduction, Oxford: Wiley-Blackwell.

Carnap, R. (1931), “Die logizistische Grundlegung der Mathematik”, Erkenntnis 2: 91-105.

Dawson, J. W. Jr. (1984), "Discussion on the Foundation of Mathematics", History and Philosophy of Logic 5(1): 111129. 
Gödel, K. (1931), "Diskussion zur Grundlegung der Mathematik am Sonntag, dem 7. Sept. 1930. Nachtrag", Erkenntnis 2: 147-151. (Versión castellana de Jesús Mosterín: "Discusión sobre la fundamentación de la matemática”, en Gödel (2006), pp. 90-93.)

Gödel, K. (2006), Obras completas (ed. por Jesús Mosterín), Madrid: Alianza, 2ª ed.

Hahn, H., Carnap, R., von Neumann, J., Scholz, H., Heyting, A., Gödel, K. y K. Reidemeister (1931), “Diskussion zur Grundlegung der Mathematik", Erkenntnis 2: 135-151.

Haller, R. (1985), "Der erste Wiener Kreis“, Erkenntnis 22(1/3): 341-358. (Reimpreso en: Haller, R., Fragen zu Wittgenstein und Aufsätze zur Österreichischen Philosophie, Amsterdam: Rodopi, 1986, pp. 89-107.)

Hempel, C. (2000), "Intellectual Autobiography - The Interview with Richard Nollan”, en Hempel, C. (2000), Science, Explanation and Rationality (comp. por J. H. Fetzer), Oxford: Oxford University Press, pp. 3-35.

Heyting, A. (1931), “Die intuitionistische Grundlegung der Mathematik”, Erkenntnis 2: 106-116.

Köhler, E. (1991), “Gödel und der Wiener Kres”, en Kruntorad, P. (con colab. de R. Haller y W. Hochkeppel) (ed.), Jour fixe der Vernunft. Der Wiener Kreis und die Folgen, Wien: Hölder-Pichler-Tempsky, pp. 127-150.

Körner, S. (1960), The Philosophy of Mathematics. An Introductory Essay, Londres: Hutchinson and Co. (Reimpresión: Nueva York: Dover, 2009. Versión castellana de Carlos Gerhard: Introducción a la filosofía de la matemática, México: Siglo Veintiuno Editores, 1967.)

Legris, J. (1996), “Carnap's Reconstruction of Intuitionistic Logic in The Logical Syntax of Language”, en Angelelli, I. y M. Cerezo (eds.), Studies on the History of Logic. Proceedings of the III. Symposium on the History of Logic, Berlin/ $\mathrm{New}$ York: Walter de Gruyter, pp. 369-375.

Mancosu, P. (1999), “Between Vienna and Berlin: The Immediate Reception of Gödel's Incompleteness Theorems”, History and Philosophy of Logic 20(1): 33-45.

Neugebauer, O. (1931), “Zur vorgriechischen Mathematik“, Erkenntnis 2: 122-133.

Pincock, C. (2011), "Philosophy of Mathematics", en Saatsi, J. y S. French (eds.), Companion to the Philosophy of Science, London: Continuum Press, pp. 314-333.

Raggio, A. R. (1990), "El cincuentenario de los Grundlagen der Mathematik de Hilbert y Bernays", Revista Latinoamericana de Filosofía 16(2): 197-211. (Reimpreso en: Raggio, A. R. (2002), Escritos completos (comp. por Alberto Moreno y Mercedes Doffi), Buenos Aires: Eudeba, pp. 391-405.)

Reid, C. (1970), Hilbert, Berlin/Heidelberg: Springer. (Reimpresión: New York: Copernicus, 1996.)

von Neumann, J. (1931), “Die formalistische Grundlegung der Mathematik”, Erkenntnis 2: 116-120.

Weyl, H. (1921), “Über die neue Grundlagenkrise der Mathematik”, Mathematische Zeitschrift 10: 39-79. 